\title{
NOTE ON A THEOREM OF AVAKUMOVIĆ
}

\author{
J. L. GELUK
}

(Communicated by J. Marshall Ash)

\begin{abstract}
A short proof is given of a result due to Avakumović. More specifically the asymptotic behavior of the solution $y(x) \rightarrow 0(x \rightarrow \infty)$ of the differential equation $y^{\prime \prime}=\phi(x) y^{\lambda}(\lambda>1)$ in case $\phi(t x) / \phi(x) \rightarrow t^{\sigma}(x \rightarrow \infty)$, $\sigma>-2$ is given.
\end{abstract}

In a paper published in 1947, Avakumovic [1] studies the asymptotic behavior of solutions $y(x) \rightarrow 0 \quad(x \rightarrow \infty)$ of the differential equation

$$
y^{\prime \prime}=\phi(x) y^{\lambda}, \quad \text { with } \lambda>1 .
$$

If $\phi$ is regularly varying with exponent $\sigma>-2$, notation $\phi \in \mathbf{R V}_{\sigma}$ (i.e., $\phi$ is measurable and eventually positive and $\phi(x y) / \phi(x) \rightarrow y^{\sigma}(x \rightarrow \infty)$ for $\left.y>0\right)$ and if $y(x)$ is a solution of (1) satisfying $y(x) \rightarrow 0 \quad(x \rightarrow \infty)$, then

$$
y(x) \sim\left[\frac{(1+\lambda+\sigma)(\sigma+2)}{(\lambda-1)^{2}}\right]^{1 /(\lambda-1)}\left\{x^{2} \phi(x)\right\}^{-1 /(\lambda-1)} \quad(x \rightarrow \infty) .
$$

The above result is generalized to the equation $y^{\prime \prime}=f(x) \phi(y)$ in three papers by Marić and Tomić [5, 6, 7]. A related paper is Omey [8].

Here we present a simple proof of the original result using the following well-known approximation result on regularly varying functions:

Lemma (see [2, Theorem 17]). Suppose $f \in \mathbf{R V}_{\alpha}$. Then there exist two functions $f_{1} \sim f_{2}$ such that $f_{1}(t) \leq f(t) \leq f_{2}(t)$ for $t \geq t_{0}$ and such that the functions $\psi_{i}(t):=\log f_{i}\left(e^{t}\right)$ are $C^{\infty}$ on a neighborhood of $\infty$ and satisfy

$$
\psi_{i}^{\prime}(\tau) \rightarrow \alpha \quad(\tau \rightarrow \infty)
$$

and

$$
\psi_{i}^{(n)}(\tau) \rightarrow 0 \quad(\tau \rightarrow \infty), n \geq 2
$$

for $i=1,2$.

Theorem. If $y$ is a bounded positive solution of the differential equation $y^{\prime \prime}=$ $\phi(x) y^{\lambda}$ with $\phi \in \mathrm{RV}_{\sigma}, \sigma>-2$, and $\lambda>1$ constant, then

$$
y \in \mathrm{RV}_{-(\sigma+2) /(\lambda-1)} \text {. }
$$

Received by the editors September 27, 1989 and, in revised form, May 16, 1990.

1980 Mathematics Subject Classification (1985 Revision). Primary 34E05. 
Proof. Substitution of $u=y^{1-\lambda}$ and $v(x)=\log u\left(e^{x}\right)$ shows that $v$ satisfies the equation

$$
v^{\prime \prime}-v^{\prime}-\beta v^{\prime 2}=-e^{\psi-v}
$$

where $\psi=\psi(x) \log \left\{(\lambda-1) e^{2 x} \phi\left(e^{x}\right)\right\}$ and $\beta=(\lambda-1)^{-1}>0$.

By the lemma, there exists $\psi_{1}$ with $\psi-\psi_{1} \rightarrow 0, \psi_{1}^{\prime} \rightarrow \sigma+2, \psi_{1}^{\prime \prime} \rightarrow 0$, and $\psi_{1} \leq \psi$ for $x$ sufficiently large. Substituting $v=\psi_{1}+c$ in (3) now gives

$$
c^{\prime \prime}-\gamma c^{\prime}-\beta c^{\prime 2}=-(1+o(1)) e^{-c}+(\sigma+2)(1+\beta \sigma+2 \beta)+o(1),
$$

with $\gamma:=\gamma(x) \rightarrow 2 \beta(\sigma+2)+1(x \rightarrow \infty)$. We claim that $c=c(x)$ tends to a finite limit as $x \rightarrow \infty$.

The following three cases are possible:

(i) $c^{\prime}>0$ for $x>x_{0}$. Then $c$ is ultimately increasing; i.e., $\lim c(x) \leq \infty$ exists. If $c(x) \rightarrow \infty$, then by (4), for $x$ sufficiently large, $\delta^{\prime}>\gamma \delta+\beta \delta^{2}>\frac{1}{2} \delta$, where $\delta:=c^{\prime}$. This implies $\delta \rightarrow \infty$ and by $(4),(-1 / \delta)^{\prime}=\delta^{\prime} / \delta^{2} \rightarrow \beta$; hence, $-1 / c^{\prime}=-1 / \delta \sim \beta x(x \rightarrow \infty)$. This contradicts the assumption $c^{\prime}>0$.

(ii) There is a sequence $x_{k} \rightarrow \infty \quad(k \rightarrow \infty)$ with $c^{\prime}\left(x_{k}\right)=0$. Assume $x_{k}$ is the sequence of all consecutive zeros of $c$. If $c^{\prime \prime}\left(x_{k}\right)<0(c$ attains its maximum in $\left.x_{k}\right)$ and $\varepsilon>0$ arbitrary, then $c\left(x_{k}\right)<-\log \{(\sigma+2)(1+\beta \sigma+2 \beta)\}$ for large $k$, by (4). Similarly, if $c^{\prime \prime}\left(x_{k}\right)>0$ we find $c\left(x_{k}\right)>-\log \{(\sigma+2)(1+\beta \sigma+2 \beta)\}$; hence, a contradiction.

(iii) $c^{\prime}<0$ for $x>x_{0}$. Then $c$ is ultimately decreasing. If $c(x) \rightarrow \infty(x \rightarrow$ $\infty)$, then, since $\psi_{1} \leq \psi$, we have, using (3),

$$
-v^{\prime \prime}+v^{\prime}+\beta v^{\prime 2}=e^{\psi-v} \geq e^{\psi_{1}-v}=e^{-c} \text {. }
$$

Hence there exists a sequence $x_{n} \rightarrow \infty(n \rightarrow \infty)$ such that $v^{\prime}\left(x_{n}\right) \rightarrow \pm \infty$. If $v^{\prime}\left(x_{n}\right) \rightarrow+\infty$, then $c^{\prime}\left(x_{n}\right) \rightarrow+\infty$; hence, a contradiction. The case $v^{\prime}\left(x_{n}\right) \rightarrow$ $-\infty$ implies $u^{\prime}\left(\exp x_{n}\right)<0$; hence, $y^{\prime}\left(\exp x_{n}\right)>0$ for $n$ sufficiently large. Since $y^{\prime \prime}>0$, this contradicts the boundedness of $y$.

This finishes the proof, since $\psi-v \rightarrow$ constant implies $x^{2} \phi(x) \sim c y^{1-\lambda}(x)$; hence, $y$ is regularly varying.

Remark. The conclusion $y \in \mathrm{RV}_{-(\sigma+2) /(\lambda-1)}$ implies that $y \rightarrow 0$. Moreover, $y^{\prime \prime}$ is regularly varying as the product of two regularly varying functions. Application of Karamata's theorem (see, e.g., [3, 4]) then gives $x^{2} y^{\prime \prime} \sim c_{0} y$ with $c_{0}=(\sigma+2)(\sigma+1+\lambda) /(\lambda-1)^{2}$. Substituting this in (1) gives (2).

\section{REFERENCES}

1. V. G. Avakumović, Sur l'équation différentielle de Thomas-Fermi, Publ. Inst. Math. (Beograd)(N. S.) 1 (1947), 101-113.

2. A. A. Balkema, J. L. Geluk, and L. de Haan, An extension of Karamata's Tauberian theorem and its connection with complementary convex functions, Quart. J. Math. Oxford Ser. (2) 30 (1979), 385-416. 
3. N. H. Bingham, C. M. Goldie, and J. L. Teugels, Regular variation, Cambridge Univ. Press, Cambridge, 1987.

4. J. L. Geluk and L. de Haan, Regular variation, extensions and Tauberian theorems, CWI tract 40, Amsterdam, 1987.

5. V. Marić and M. Tomić, Asymptotic properties of solutions of the equation $y^{\prime \prime}=f(x) \phi(y)$, Math. Z. 149 (1976), 261-266.

6. __ Regular variation and asymptotic properties of solutions of nonlinear differential equations, Publ. Inst. Math. (Beograd) (N. S.) 21 (1977), 119-129.

7. __ Asmptotic properties of solutions of a generalized Thomas-Fermi equation, J. Differential Equations 35 (1980), 36-44.

8. E. Omey, Regular variation and its applications to second order linear differential equations, Bull. Soc. Math. Belg. Sér. B 33 (1981), 207-229.

EConometric Institute, ERASmus University Rotterdam, P.O. Box 1738, 3000 DR RotTERDAM, THE NETHERLANDS 